Number of pages: 26

Number of references: 16

Number of figures: 15

\title{
Measuring vibration-induced variations in pressures between the human body and a seat
}

Authors:

Chi Liu and Michael J Griffin

Affiliations

Human Factors Research Unit

Institute of Sound and Vibration Research

University of Southampton

Southampton SO17 1BJ

England

Correspondence address:

Dr. Chi Liu

Telephone: (+44) 01912088657

E-mail: liuchi0511@gmail.com

This manuscript is submitted as an Original Article.

There is no known conflict of interest in respect of this paper. 


\section{Highlights}

- Sitting comfort is influenced by the distribution of static and dynamic pressures

- Measuring the distribution of dynamic pressures caused by vibration is currently challenging

- A method of calibrating pressure sensors in a pressure mat is proposed

- Recommendations are made for measuring dynamic pressures in vibration environments 


\section{Abstract}

When measuring total dynamic forces between the human body and seats during vibration, variations in force over the buttocks and the thighs have previously been ignored. In this study, sensors in a pressure mat were calibrated by applying static and dynamic pressures through foam and calculating transfer functions between the applied pressures and the outputs of the sensors. This provided static calibration of individual pressure sensors and dynamic calibration of groups of pressure sensors. Measurements of dynamic pressures were used to calculate the vertical forces over the ischial tuberosities, middle thighs, and front thighs and compare with similar measurements obtained by another method. Dynamic calibration of the pressure mat reduced errors in the total dynamic force to $10 \%$ at frequencies less than $5 \mathrm{~Hz}$ and $30 \%$ at frequencies between 5 to $15 \mathrm{~Hz}$. Recommendations are offered for measuring dynamic pressures between the human body and seats in vibration environments.

Relevance to industry: Dynamic pressure distributions at seat interfaces influence sitting comfort during whole-body vibration. Understanding of the dynamic performance of pressure sensors and recommendations on calibrating and using pressure sensors in vibration environments can assist seat design and other situations where dynamic pressure measurements are of interest.

Keywords: static and dynamic human-seat interface pressures; whole-body vibration; pressure mat calibration. 


\section{Introduction}

In static conditions, sitting comfort is influenced by static pressures between the human body and the seat (Ebe and Griffin, 2001). In vibration conditions, dynamic forces over the seat surface contribute to the discomfort caused by whole-body vibration (Ebe and Griffin, 2000a,b; Zhou and Griffin, 2014). Although the distribution of dynamic pressures between the human body and a seat during whole-body vibration contribute to discomfort, there are doubts about the accuracy of measurements of dynamic pressures and the calibration of pressure sensors.

The total forces acting on the human body sitting on a seat have been measured to quantify the dynamic response of the seated human body while exposed to whole-body vibration (e.g., Fairley and Griffin, 1989). Although total forces reflect the overall properties of the body, such as the principal resonance and the effective stiffness and damping, the variation in dynamic response over the contact area of the buttocks (including the ischial tuberosities and the thighs) is ignored. If the distribution of dynamic pressures between the human body and a seat could be accurately measured during vibration they would be expected to show a large variation.

The dynamic pressures at the human-seat interface have been measured over both a rigid seat (e.g., Wu et al., 1998) and a compliant seat (Wu et al., 1999). Pressure measurements have also been used to derive the apparent mass of the body (i.e., the transfer function between the dynamic force over the seat and the acceleration of the seat) when sitting on a compliant seat (Hinz et al., 2006; Dewangan et al., 2013). Plantar pressures have been measured to derive the apparent mass of the human body standing in different postures while exposed to vertical vibration (Tarabini et al., 2013).

Flexible thin-film pressure sensors have been used in the above studies. There are currently two sensor types: capacitive sensors (as used by Wu et al., 1998, 1999; Hinz et al., 1998; Tarabini et al., 2013) and resistive sensors (as used by Dewangan et al., 2013). In capacitive sensors, the distance between the two parallel plates of a condenser (i.e., the sensor) changes with the applied pressure, resulting in a change in the capacitance. In a resistive sensor, the resistance of the sensor is affected by the load. Both types of sensor have been reported to have non-ideal performance for both static measures (Paikowsky and Hajduk, 1997; Arndt, 2003; Palmer et al., 2009; Saggin et al., 2013) and dynamic measures (Dewangan et al., 2013; Tarabini et al., 2013; Saggin et al., 2013).

When measuring static pressure with either type of sensor, the pressure signals have been found to increase over time during a constant static load (Paikowsky and Hajduk, 1997; Arndt, 
2003; Palmer et al., 2009; Saggin et al., 2013). The extent of the increase has varied between studies (Paikowsky and Hajduk, 1997; Arndt, 2003; Saggin et al., 2013) and has been reported to be independent of load but dependent on temperature (Saggin et al., 2013).

When measuring dynamic pressures with either type of sensor, the forces calculated from the contact area and the measured pressures have been found to be lower than the applied forces, with greater underestimation at higher frequencies (Dewangan et al., 2013; Tarabini et al., 2013; Saggin et al., 2013). The underestimation is suggested to arise from the viscoelastic behaviour of the material of the sensors (Tarabini et al., 2013; Saggin et al., 2013) and the low sampling rates of the system (Dewangan et al., 2013).

The static calibration of pressure sensors is performed by applying static pressures over the sensors for a period of time (e.g., Palmer et al., 2009). Since the pressure signals increase over time during a constant static load, the coefficient that relates the digital outputs of a sensor to the applied pressure is time-dependent. In measurements, the sensor is loaded for a period of time before recording and the coefficient that has been obtained for the same period of static loading during calibration is used to derive the pressures from the sensor outputs.

The dynamic calibration of pressure sensors involves measuring transfer functions between the outputs of the pressure sensors and the applied dynamic loads, so the transfer functions can be used to correct the sensor outputs measured in experiments (e.g., Tarabini et al., 2013; Saggin et al., 2013; Dewangan et al., 2013). Dewangan et al. (2013) measured dynamic forces at the interface between a seated human body and a rigid seat using a force plate and also estimated the forces from pressures measured by a pressure mat. Both forces were used to calculate the vertical apparent mass of the seated human body and the ratio between the two apparent masses in the frequency domain was used to correct the apparent mass obtained using the pressure mat. In other studies, forces have been applied to individual sensing units of a pressure mat and transfer functions between the applied forces and the measured forces used to correct pressures measured when using the pressure mat (Tarabini et al., 2013; Saggin et al., 2013).

The dynamic calibration method used by Dewangan et al. (2013) is based on the total forces, and thus the obtained relationship between the sensor outputs and the applied loads is an average over all sensing units. If there is variation in sensitivity between sensing units, the relationship would depend on the distribution of the load over the pressure mat and not applicable for all loading conditions, so the relationship differed between subjects and depended on vibration magnitude (Dewangan et al., 2013). Although dynamic calibration of each sensing unit seems optimal, as reported by Saggin et al. (2013), this is difficult and time consuming for pressure mats containing thousands of sensing units. It would be more practical 
to undertake dynamic calibration by applying evenly distributed loads over groups of sensing units, but it is unknown whether this will reduce errors in either the total forces or the forces indicated by individual sensors.

Analogue signals from hundreds or thousands of sensing units on a pressure mat are scanned in sequence and then digitised. This introduces sampling delays between sensing units which increase with increasing number of sensors. There are consequent errors in measurements of dynamic pressure that depend on the scan rate and the frequency of the applied vibration.

The objective of this study was to define a method of quantifying the static and dynamic performance of a resistive pressure sensor. This involved investigating the causes of errors and defining a calibration method with controlled dynamic forces. Errors when using a calibrated system to measure dynamic pressures at the human-seat interface during wholebody vertical vibration were then investigated. It was hypothesised that the calibration method would reduce errors when using a pressure mat to measure the total dynamic forces between the human body and a seat during vertical vibration.

\section{Materials and methods}

\subsection{Pressure mapping system}

The study investigated a resistive pressure mapping system consisting of a pressure mat and a data acquisition system. The pressure mat had a sensing matrix located between two polymeric films (Figure 1). The sensing matrix consisted of two layers of conductive strips, with one layer distributed in rows at equal intervals and the other in columns at equal intervals. The conductive strips were covered by a layer of semi-conductive material with a rough surface. With pressure applied to the polymeric film, the upper conductor is forced into contact with the lower conductor at the intersections, forming a sensing unit and causing deformation of the rough surfaces. Such deformation increases the contact area and reduces the resistance of the sensing unit. The pressure sensor had 2016 sensing units (42 rows and 48 columns) with a total sensing area of $0.2081 \mathrm{~m}^{2}$ (width: $487.68 \mathrm{~mm}$; depth: $426.72 \mathrm{~mm}$ ). 


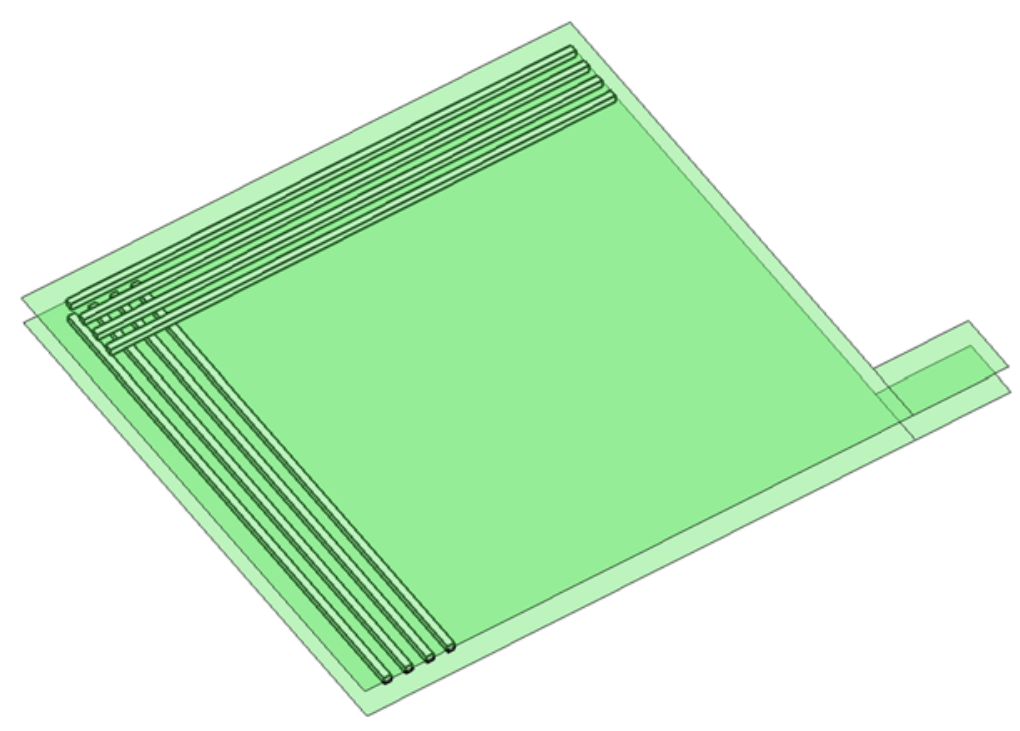

Figure 1 Schematic of structure of the resistive pressure sensor.

The acquisition system measured the resistance at each sensing unit in sequence. During acquisition, a selected column was excited by a non-zero voltage, with all the 42 sensing units on the excited column brought to the same voltage, while other columns were grounded. A multiplexor then connected each row of the mat in sequence to read the voltage that depended on the resistance of each sensing unit. After reading all the sensing units on the selected column, the reading of rows in the next column was commenced after a delay of about 20 microseconds. The maximum sampling rate was 100 frames per second, where one frame contained pressure readings from all 2016 sensing units.

Each sensor can measure up to $207 \mathrm{kPa}$ (30 psi; 2 bar). There were 30 pressure ranges with the digital output automatically scaled to the selected pressure range. The digital output of a single sensing unit was an integer number between 0 and 255 , so the resolution was the pressure range divided by 256 . The pressure range used for the measurements in Section 2.2 was determined so that it would include the maximum pressure over the contact area required for the measurements in Section 2.3.

\subsection{Static and dynamic calibration}

\subsubsection{Equipment}

An indenter rig was used to apply controlled loads over the sensor (Figure 2). The indenter rig had a steel frame mounted over an electro-dynamic shaker (Ling V860). A flat horizontal metal plate was secured to the indenter head which was driven by a motor to provide vertical 
compression forces. An RDP MCL DC load cell measured the static force and a Kistler 9347B load cell measured the dynamic force above the indenter head.

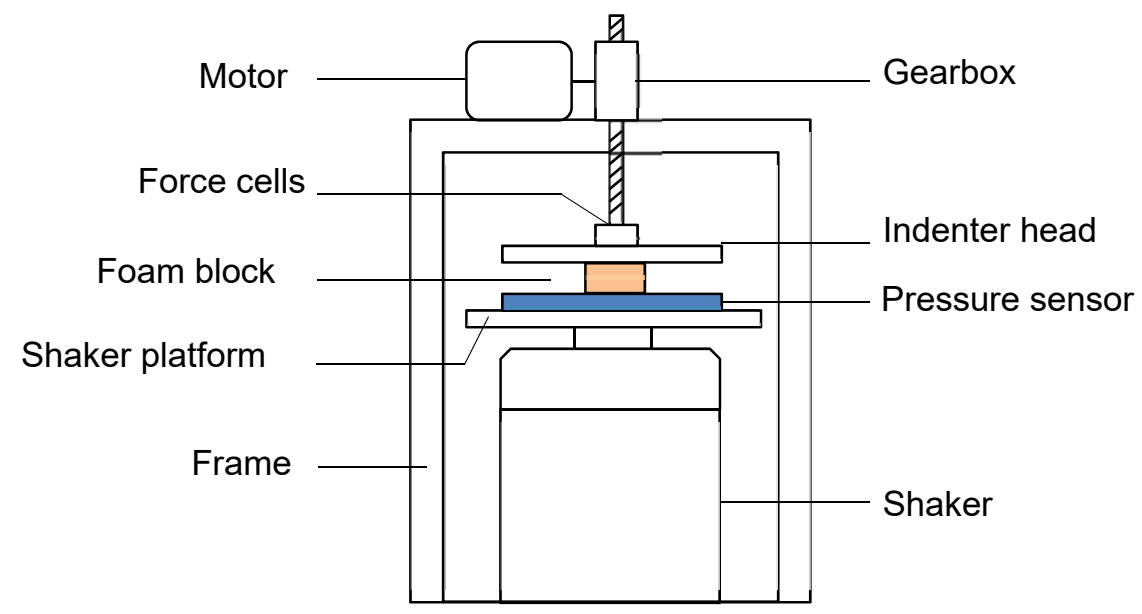

Figure 2 The indenter rig used for producing dynamic loads to the pressure sensor.

\subsubsection{Static performance}

The pressure sensor was placed on the rigid flat platform of the shaker (which did not vibrate). A polyurethane foam layer $(71 \mathrm{~mm}$ by $61 \mathrm{~mm}$ by $5 \mathrm{~mm}$ ) covered 42 sensing units at the centre area of the pressure mat. The foam had an open-cell structure with a density of about $50 \mathrm{~kg} / \mathrm{m}^{3}$ (estimated). Each of three masses $(3.8,7.2$, and $10.7 \mathrm{~kg}$ ) rested on the foam layer for 30 minutes. The pressure sensor was left unloaded for 5 minutes between each load condition. The pressure signals were acquired at 5 frames per second.

\subsubsection{Static calibration}

To perform static calibration, a rectangular foam block (173 mm by $132 \mathrm{~mm}$ by $60 \mathrm{~mm}$ ) was placed over 221 sensing units of the pressure mat (Figure 2). The foam had an open-cell structure with a density of about $50 \mathrm{~kg} / \mathrm{m}^{3}$. The indenter head compressed the foam block at a constant velocity of $0.5 \mathrm{~mm} / \mathrm{s}$ until the average digital output of the loaded sensing units reached about 50,100 , or 180 (the full range of the digital output of the pressure sensor was 255, see Section 2.1). The total force over the contact area corresponded to approximately 150,440 , and $600 \mathrm{~N}$. The indenter position was then maintained for 10 minutes. Recordings of force and pressure commenced before compressing the foam. The pressure sensor was unloaded for 5 minutes between each measurement.

The force signals from the RDP MCL DC load cell of the indenter rig were sampled at 5 samples per second by an HVLab system via $100-\mathrm{Hz}$ anti-aliasing filters. The pressure data were acquired at 5 frames per second. 
The pressures indicated by the pressure mat were compared with the mat rotated on the platform in the horizontal plane by 90 and 180 degrees. Relative to the position of the indenter rig, the pattern in the pressure distributions rotated with the rotation of the mat (Figure 3 ), suggesting the pattern mainly arises from variations between the sensing units, not from the indenter rig or the foam (e.g., the parallelism between the indenter head and platform, the composition and density distribution inside the foam, etc.).
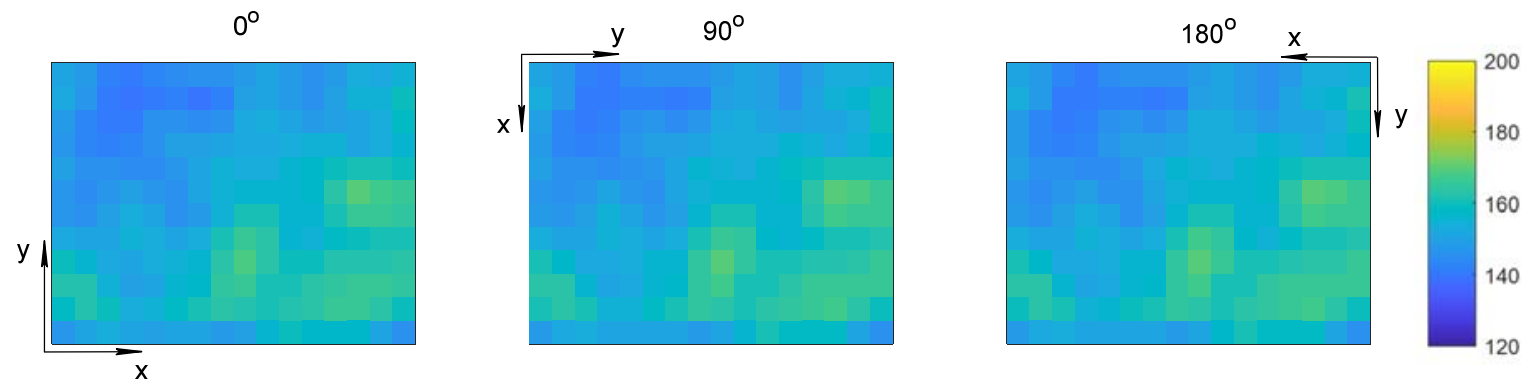

Figure 3 Comparison of digital outputs during a static pressure load with the pressure mat rotated in the horizontal plane by $90^{\circ}$ and $180^{\circ}$; coordinates referred to the position of the indenter rig; colour bar indicates the raw digital output of the pressure sensor which is an integer number between 0 and 255.

The force applied to an individual sensing unit was calculated by dividing the force measured by the force cell by the number of the loaded sensing units, assuming the pressure beneath the foam was uniformly distributed by compressing the foam vertically with the horizontal flat indenter head. For each of the three applied loads, the ratio between the digital output of a sensing unit and the corresponding applied force was obtained during compression at 60, 120, 300 , and $600 \mathrm{~s}$. At each loading duration, the ratios obtained with the three applied forces were fitted linearly with straight lines passing through the origin. The slope of each line was used as the gain to convert the digital output of the corresponding sensing unit into force.

\subsubsection{Dynamic calibration}

A rectangular foam block ( $203 \mathrm{~mm}$ by $203 \mathrm{~mm}$ by $60 \mathrm{~mm}$ ) was placed over 400 sensing units of the pressure mat on the top plate of the shaker (Figure 2). The foam had an open-cell structure with a density of $49 \mathrm{~kg} / \mathrm{m}^{3}$. The indenter head compressed the foam at a constant velocity of $0.5 \mathrm{~mm} / \mathrm{s}$ until the average digital reading of the sensing units reached about 60 , $90,120,150,180$ (the full range of the pressure sensor was 255 , see Section 2.1). The position of the indenter head was then fixed and the pressure mat was loaded for 5 minutes before presenting vibration. The shaker then presented a 120-s sine sweep from 1 to $18 \mathrm{~Hz}$ with a constant peak-to-peak displacement of $1 \mathrm{~mm}$. For the five preload forces, the peak-to-peak magnitudes of the corresponding dynamic forces acting on a sensing unit were about 0.10 , 
$0.11,0.16,0.19,0.33 \mathrm{~N}$. The pressure sensor was left unloaded for 5 minutes between each load condition.

The applied forces were measured above the indenter head, as shown in Figure 2. The forces were regarded equal to the forces beneath the foam considering the small mass of the foam (about $0.1 \mathrm{~kg}$ ) and the small displacement of the vibration. The dynamic stiffness of the foam block (transfer function between the displacement and force) measured with the sine sweep showed an approximately flat modulus with zero phase (Figure 4), indicating that the foam performed as a spring between 1 and $18 \mathrm{~Hz}$ and that the dynamic force was approximately constant over this range of frequencies.

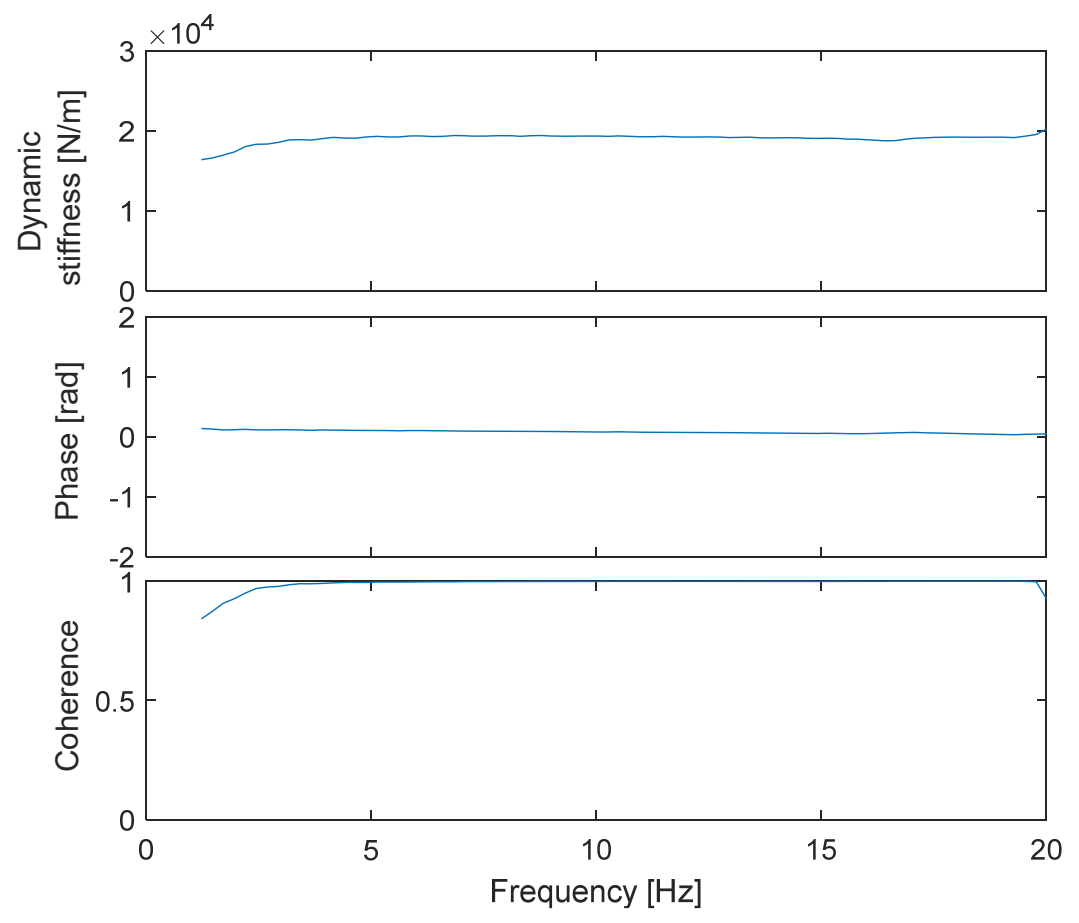

Figure 4 Dynamic stiffness of the foam measured with sine sweep excitation; the average digital reading of the sensing units due to the static preload was 150.

The applied force was measured using the Kistler 9347B force cell of the indenter rig, sampled at 512 samples per second by the HVLab system via $100-\mathrm{Hz}$ anti-aliasing filters. The pressure data were sampled at 100 frames per second and later resampled at 512 samples per second using the HVLab data analysis toolbox. The calibration gain during compression at $300 \mathrm{~s}$ in the static calibration were used to convert the digital outputs into force $(\mathrm{N})$.

Transfer functions, $H_{1}(f)$, were calculated between the force measured with the Kistler force cell, $F_{0}(f)$, and the sum of the forces indicated by the pressure sensors, $F_{1}(f)$ :

$$
H_{1}(f)=F_{0}(f) / F_{1}(f)
$$


The forces acting on individual sensing units, $F_{0 i}(f)$, were estimated by dividing the force measured with the Kistler force cell by the number of loaded sensing units (i.e., 400). Transfer functions, $H_{2 i}(f)$, were calculated between the estimated individual forces, $F_{0 i}(f)$, and the measured individual forces from each sensing unit, $F_{1 i}(f)$ :

$$
H_{2 i}(f)=F_{0 i}(f) / F_{1 i}(f)
$$

The above transfer functions were calculated from the ratio of the power spectral density of the output, $G_{o o}(f)$, to the cross spectral density between the output and the input, $G_{\mathrm{oi}}(f)$ (i.e., using $\mathrm{H} 2$ estimation because of the greater noise in the input):

$$
H(f)=G_{o o}(f) / G_{o i}(f)
$$

Relationships between sensor readings (e.g., digital outputs) and loading forces will usually be expressed in terms of 'sensor gain' when calibrating a pressure mat, and so the relationships are presented here with the sensor readings on the abscissa and forces on the ordinate, showing the force per digital output.

\subsection{Testing the effectiveness of the calibration}

\subsubsection{Equipment}

A rigid seat was secured to the platform of a 1-m vertical shaker. A Silicon Designs 2260-002 accelerometer was secured to the seat frame to measure the seat vibration. A force plate designed to measure separately the forces beneath the ischial tuberosities, the middle thighs, and the front thighs during vertical vibration was mounted to the frame of the rigid seat (Liu et al., 2017). The pressure sensor was placed on top of the force plate (Figure 5). The sensitivity of the pressure sensor was chosen so that the pressure range encompassed the maximum pressure at the interface during vibration. 

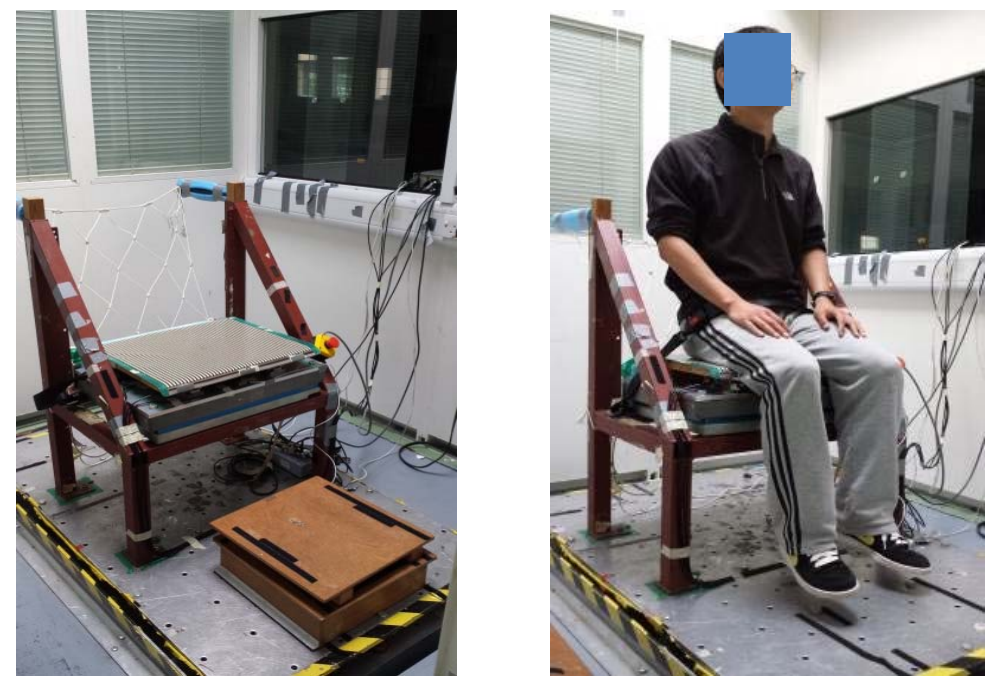

Figure 5 Experimental setup for measuring the pressures between a subject and a rigid seat during vertical vibration. Left: pressure mat over the seat surface; right: subject sitting with loose lap belt and no backrest contact and no foot support.

\subsubsection{Subject and stimulus}

A male subject $(170 \mathrm{~cm}, 65 \mathrm{~kg}$ ) sat on the rigid seat with a normal upright sitting posture and his feet unsupported (Figure 5). The subject was exposed to vertical random vibration with an approximately flat acceleration spectrum between 0.5 to $15 \mathrm{~Hz}$ at $1.0 \mathrm{~ms}^{-2}$ r.m.s. with a duration of $60 \mathrm{~s}$.

The experiment was approved by the Ethics Committee of the Faculty of Engineering and the Environment at the University of Southampton (approval number 7931).

\subsubsection{Analysis}

Transfer functions were calculated between the total force beneath each of three areas (i.e., the ischial tuberosities, the middle thighs, and the front thighs) and the vertical acceleration of the seat. These transfer functions are referred to as the apparent masses at the corresponding areas, consistent with Liu et al. (2017).

The total forces indicated by the pressure sensors for each area were obtained in two ways. The force outputs from all the loaded sensing units (after static calibration) were summed and then filtered by the transfer function, $H_{1}(f)$ (i.e., average dynamic calibration). Additionally, the output from each individual sensing unit was filtered by its transfer function, $H_{2 i}(f)$ (i.e., individual dynamic calibration) and the forces then summed. 


\section{Results}

\subsection{Static performance}

With all three loads, the digital outputs of all the loaded sensing units increased over time. The responses of an example sensing unit (identified as $[23,23]$, the unit at the $23^{\text {rd }}$ row and the $23^{\text {rd }}$ column) are shown in Figure $6 a$. When the digital outputs were normalised to the output measured at $20 \mathrm{~s}$, all the sensing units showed similar responses for all three loads, as the example shown in Figure 6b, suggesting there was little effect of load on the timedependencies of the sensing units.
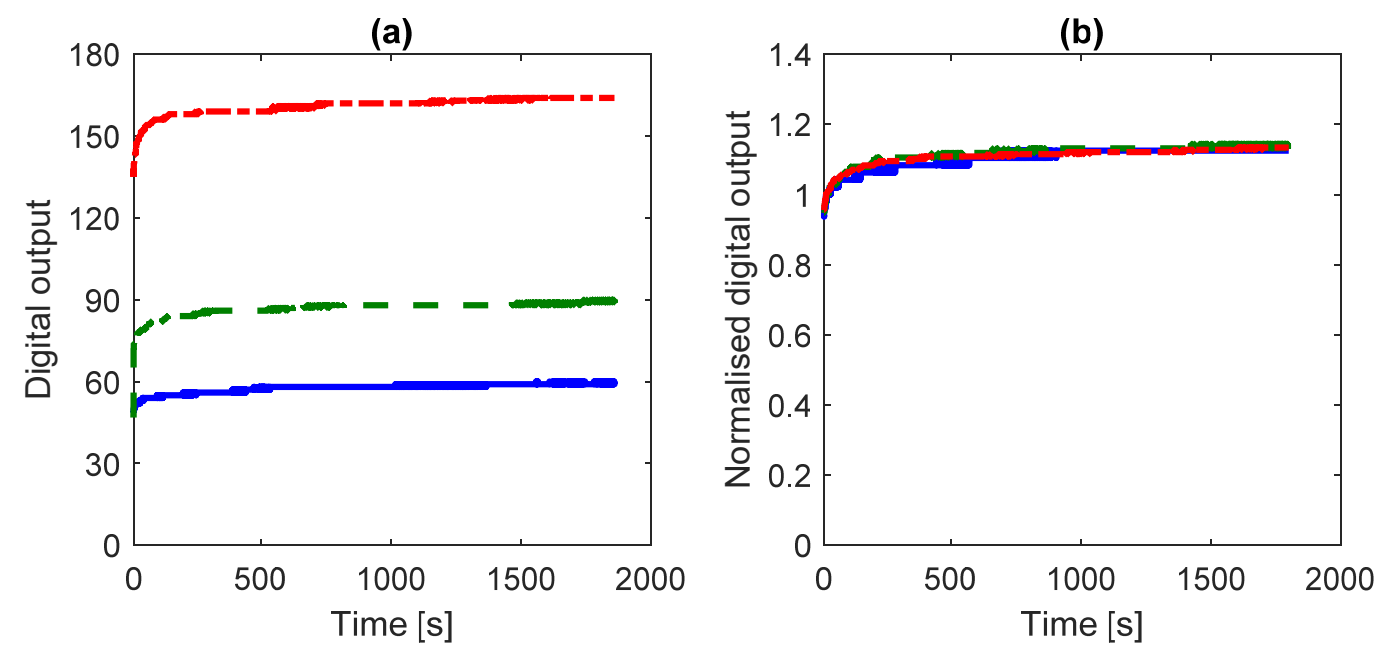

Figure 6 Digital outputs of one sensing unit $(23,23)$ with three static loads: - $3.8 \mathrm{~kg}$; - $-7.2 \mathrm{~kg}$; ---.- $10.7 \mathrm{~kg}$; (a): raw digital outputs; (b): each digital output normalised to the digital output at $20 \mathrm{~s}$.

\subsection{Static calibration}

Relationships between digital outputs and loaded forces (150, 440, and $600 \mathrm{~N}$ over the area, equivalent to $0.7,2.0$, and $2.7 \mathrm{~N}$ on individual units) obtained with the indenter rig are close to linear for each reading time (i.e., 60, 120, 300 and 600 s). Such linear relationships were found for all sensing units, with an example shown for an individual sensing unit in Figure 7.

The relationships between digital outputs and loaded forces obtained using the indenter rig were compared with those obtained when using a pneumatic calibration device recommended by the manufacturer of the pressure sensing system (Figure 7). The pneumatic device consisted a vacuum pump and a flat and flexible bladder housing two flat plates. During calibration, the pressure mat was placed between the plates and the pressure inside the 
bladder was reduced and maintained by the vacuum pump. The pressure mat was loaded by the difference in pressure between the atmosphere and the reduced pressure inside the bladder. For all sensing units, similar relationships were obtained with the indenter and the pneumatic calibration device.

The relationships between digital outputs and loaded forces obtained with the indenter rig at $300 \mathrm{~s}$ were fitted with a line passing through the origin for each loaded sensing unit. The slope of the line was used as the gain ( $\mathrm{N}$ per digital output) to convert the digital output into force. Among the 221 loaded units, the gain varied between 0.013 and $0.021 \mathrm{~N}$ with a mean value $0.017 \mathrm{~N}$ and standard deviation $0.0016 \mathrm{~N}$. Variations in gain seemed independent of location.

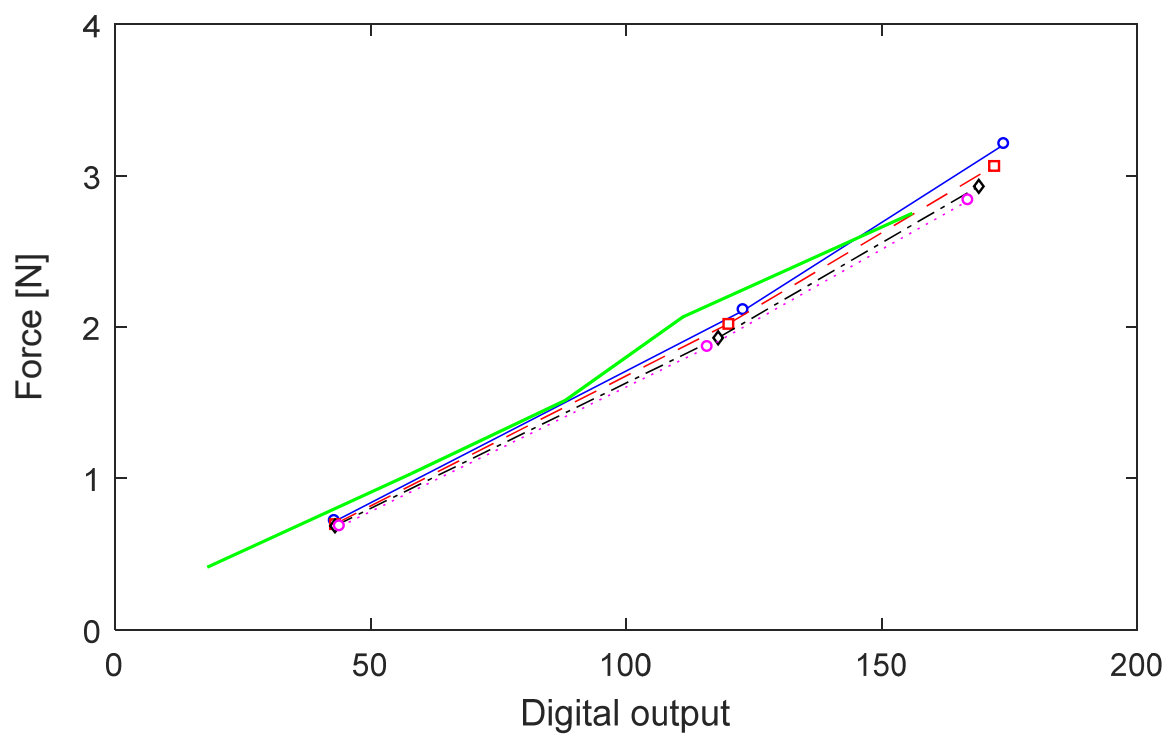

Figure 7 Relationships between digital outputs and applied forces for one sensing unit (23, 23): - - reading at $60 \mathrm{~s} ;--\square--$ reading at $120 \mathrm{~s} ;-\cdot \diamond \cdot-$ reading at $300 \mathrm{~s} ; \cdots \circ \cdots$ reading at $600 \mathrm{~s} ;-$ calibration using a pneumatic device; for clarity, only 42 of 221 loaded sensing units are shown. All sensing units showed similar relationships.

\subsection{Dynamic calibration}

Transfer functions, $H_{1}(f)$, between the total force measured by the load cell and the total force summed over 400 pressure sensors are shown for different preloads in Figure 8 . The moduli of the transfer functions were greater than unity at all frequencies and for all loads, indicating the applied force (numerator in Equation (1)) was greater than the measured force (denominator in Equation (1)) and thus the dynamic force was underestimated by the pressure sensors. With all five loads, the underestimation was greater at higher frequencies and reduced as the load increased. Depending on the load, the error was in the range $30 \%$ to $35 \%$ 
at $1 \mathrm{~Hz}$ and $24 \%$ to $41 \%$ at $15 \mathrm{~Hz}$. The error is expected to depend on the rough surfaces of the resistive sensor and will differ with different types of pressure sensor.

The phase between the total force measured by the load cell and the total force summed over 400 pressure sensors is not shown because the measurements of the two forces were not synchronised.

With all five loads, the transfer functions, $H_{2}(f)$, between the force at each sensing unit (estimated by dividing the force measured with the load cell by the number of loaded units, i.e., 400) and the corresponding force measured with each sensing unit depended on the location of contact with the foam. The mean moduli of the transfer functions of the units at the centre of the foam, around the centre, and at the edge of the foam obtained with the preload producing an average digital output of 180, are shown in Figure 9. At frequencies less than 2 $\mathrm{Hz}$, the mean moduli for sensing units at the centre were greater than those at the other locations. At all frequencies, the mean moduli for sensing units at the edge of the foam were lower than those at or around the centre.
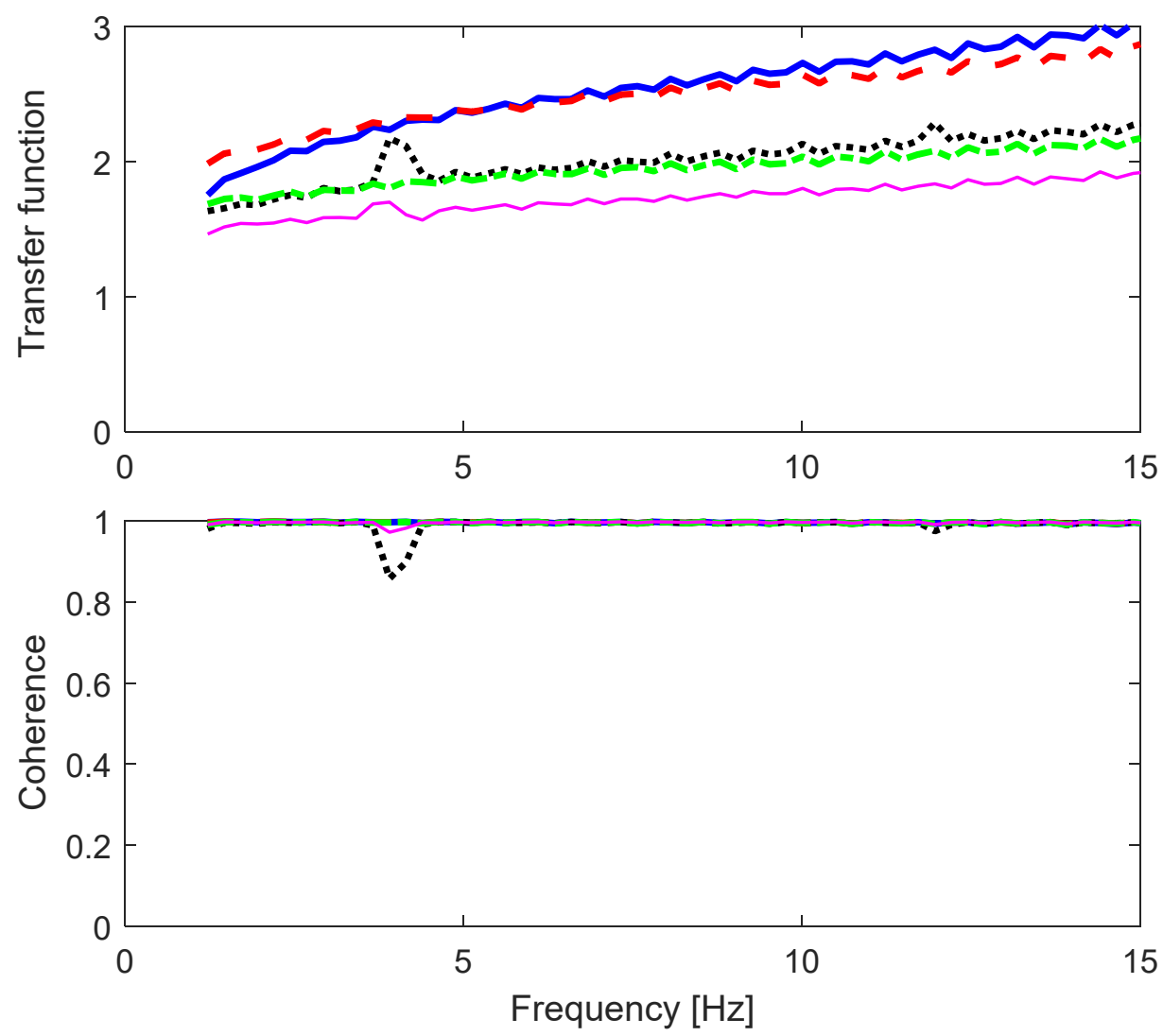

Figure 8 Transfer functions between the force measured from the load cell and the force summed from the pressure sensors (i.e. $\left.H_{1}(f)\right)$ : — case of preload producing average digital output of 60 , referred to as 'preload of 60 '; - - preload of $90 ; \cdot \cdot . \cdot$ preload of 120 ; -"-"- 
preload of 150; — preload of 180. Greater digital output corresponds to greater preload and greater dynamic forces, see Section 2.2.3.

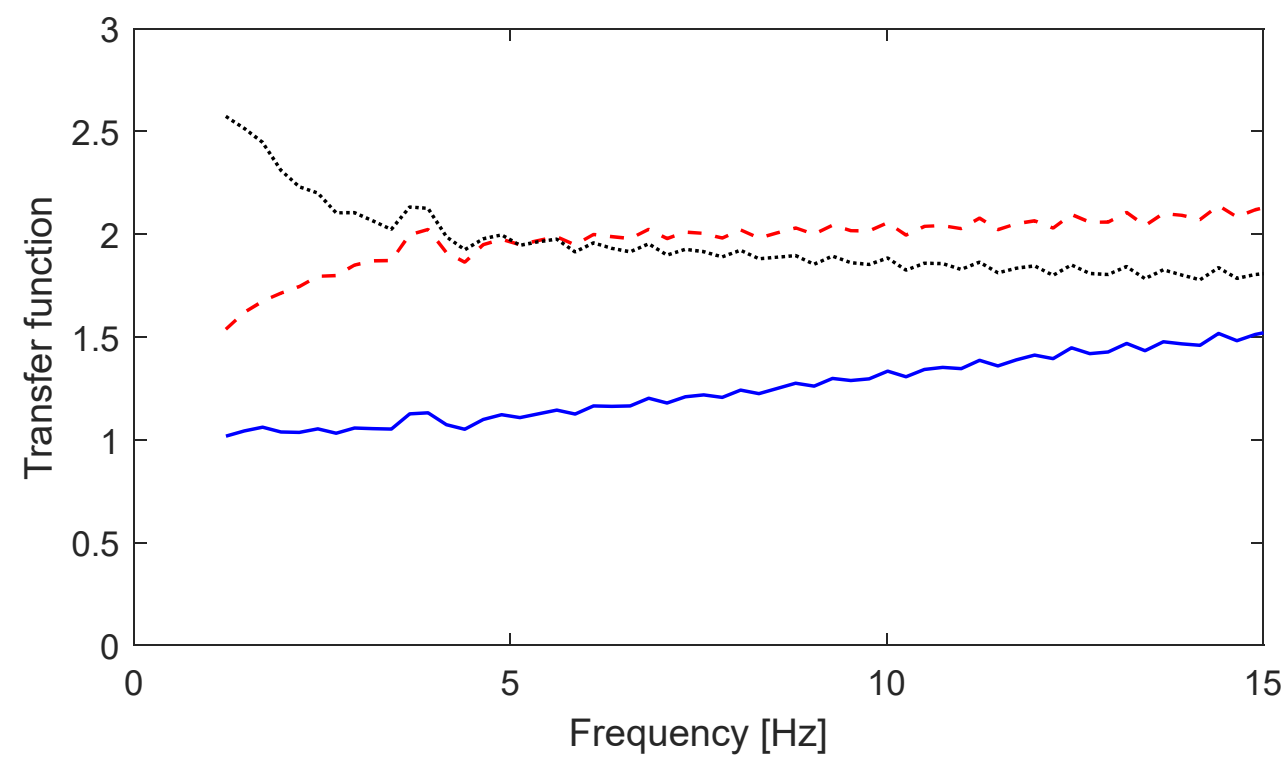

Figure 9 Mean moduli of transfer functions between the applied force and the force indicated by the sensing units (i.e. $H_{2}(f)$ ): $\cdots \cdots . .$. units located at the centre of the contact area; - - - units around the centre of the contact area; _- units at the edge of the contact area. The transfer functions are for the preload that produces an average digital output of 180 .

\subsection{Testing the effectiveness of the calibration}

Without dynamic calibration, the frequency dependence of the apparent masses at the ischial tuberosities, the middle thighs, and the front thighs was correctly reflected by the pressure sensors, but with an underestimation at all frequencies (Figure 10). The error was about $20 \%$ around $1 \mathrm{~Hz}$ and about $60 \%$ around $15 \mathrm{~Hz}$ (Figure 11). The apparent mass measured beneath the middle thighs showed greater errors than those at the other locations. Phase is not shown because the measurements of pressure and acceleration at the seat were not synchronised.

The application of either average dynamic calibration or individual dynamic calibration reduced the underestimation to less than $10 \%$ at frequencies less than $5 \mathrm{~Hz}$ and less than $30 \%$ at frequencies between 5 and $15 \mathrm{~Hz}$ (Figure 11). The calibrated dynamic force beneath the middle thighs showed greater errors than the calibrated forces at other locations. 


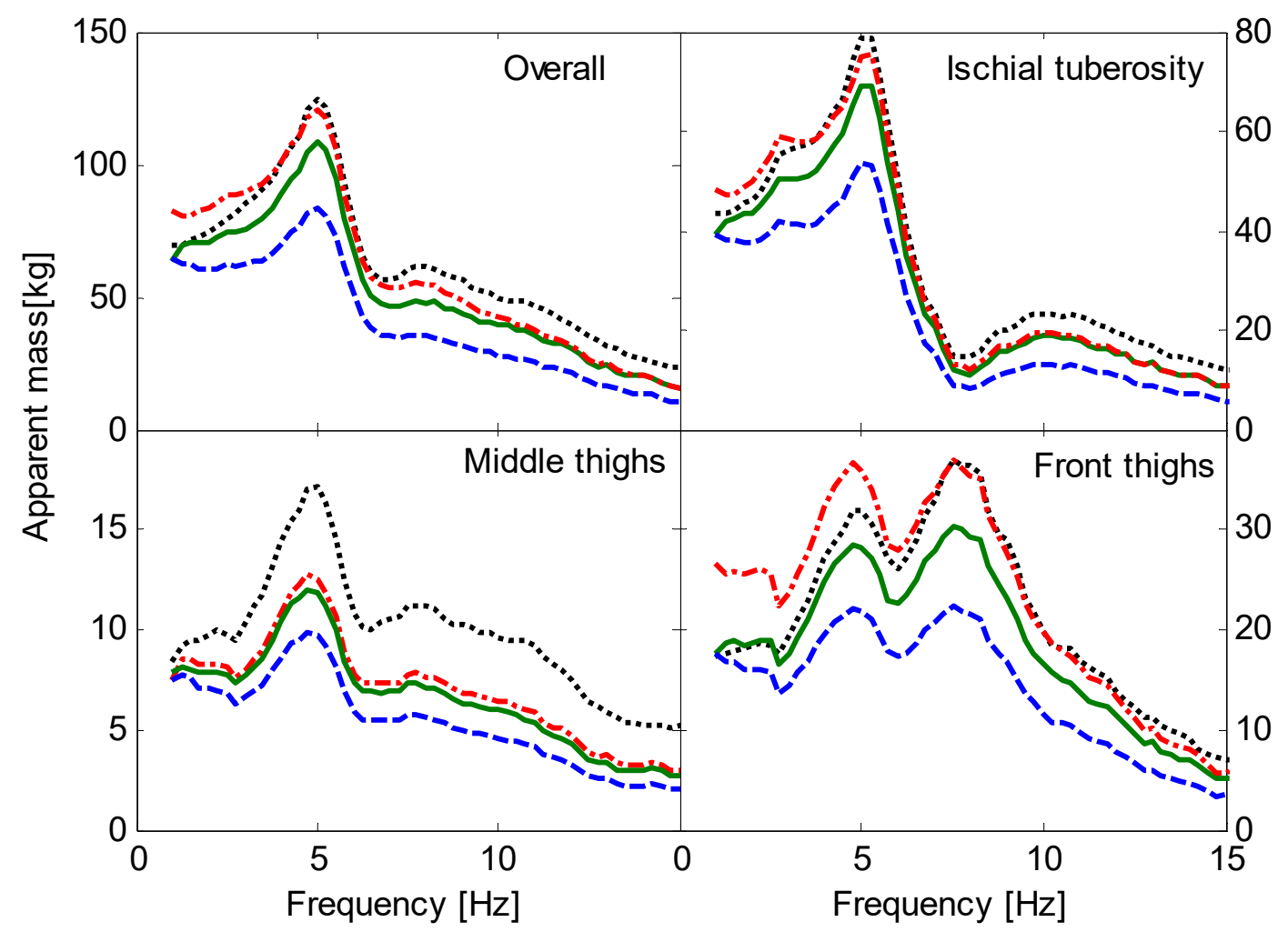

Figure 10 Overall apparent mass and the apparent masses measured at the three locations with the force plate and with the pressure sensors: $\cdots \cdots \cdot . \cdot$ measured with the force plate; - - measured with the pressure sensors without dynamic calibration; __ measured with the pressure sensors with average dynamic calibration; -.--- measured with the pressure sensors with individual dynamic calibration.

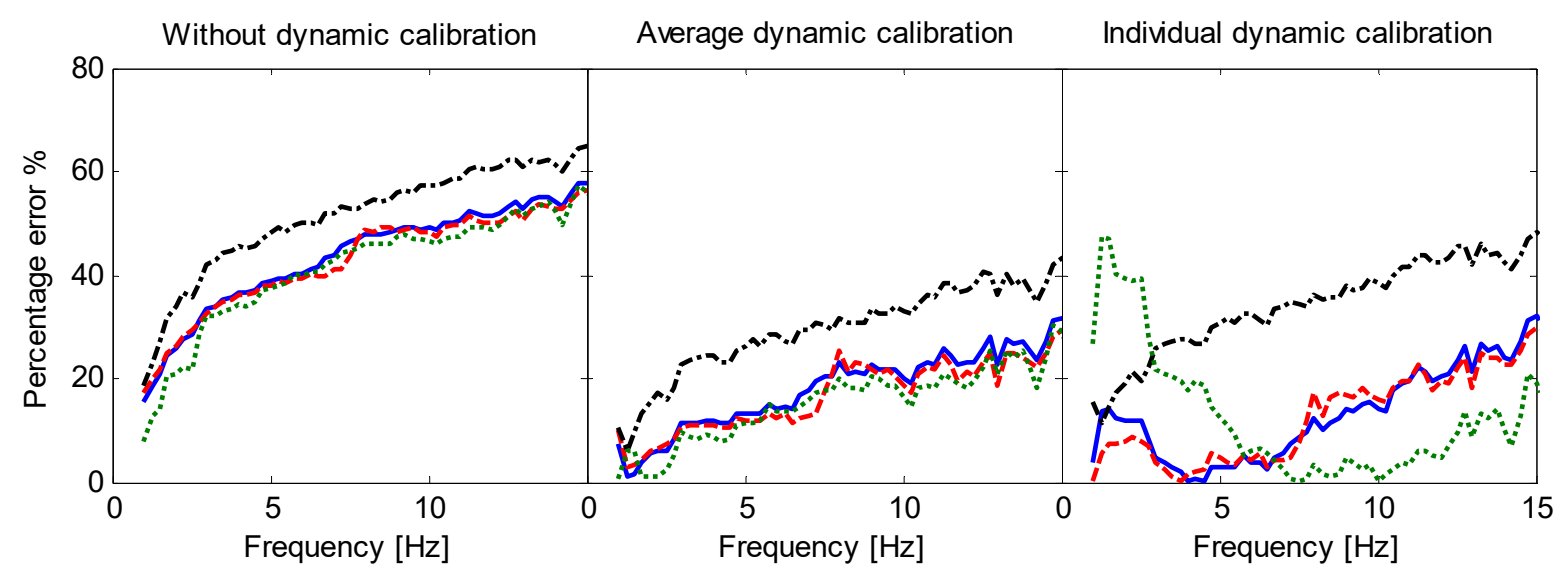

Figure 11 Percentage errors in the measured forces when using the pressure sensors with and without dynamic calibration; _ errors in the overall force; - - - errors in the force measured beneath the ischial tuberosities; -.--- errors in the force measured beneath the middle thighs; ;....... errors in the force measured beneath the front thighs. 


\section{Discussion}

\subsection{Static performance and static calibration}

With a constant load, readings from the resistive pressure sensors increased over 30 minutes with rapid increase in the first minute and only small increases subsequently (Figure 6), consistent with previous findings (Paikowsky and Hajduk, 1997; Arndt, 2003; Palmer et al., 2009; Saggin et al., 2013). The increase is similar to the creep behaviour of a viscoelastic material. It seems that with a constant load, the deformation between the two contacting rough surfaces of the upper and lower conductors increased, increasing the area of contact and decreasing sensor resistance, suggesting increased pressure.

Static calibration using the indenter indicated similar sensor sensitivity to that obtained using pneumatic pressure (Figure 7). This suggests that compressing the foam with the indenter distributed pressures evenly over the sensor mat.

\subsection{Factors affecting dynamic performance}

\subsubsection{Viscoelastic behaviour}

Dynamic loads were underestimated by the pressure sensor (Figure 8), with the underestimation broadly similar to that reported previously (Hinz, et al., 2006; Dewangan et al., 2013). At $5 \mathrm{~Hz}$, the error was about $38 \%$ in the current study but $25 \%$ in Hinz et al. (2006) and $35 \%$ in Dewangan et al. (2013). The difference may be due to the use of different types of sensor and the condition of the rough surfaces of the resistive sensor.

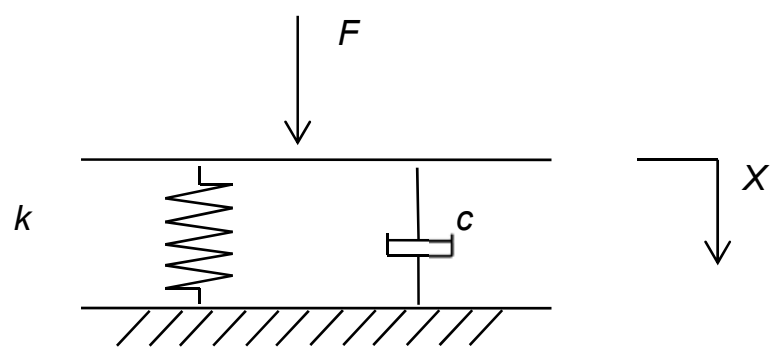

Figure $12 \mathrm{~A}$ Maxwell model of the effect of viscoelastic behaviour on the dynamic performance of the pressure sensor.

Underestimation of dynamic forces may be attributed to viscoelastic properties, illustrated with a Maxwell viscoelastic model in Figure 12. The two massless plates of the model represent the two layers of a sensing unit. The displacement, $X$, of the top plate that represents the 
deformation between the two contacting rough surfaces, determines the pressure reading. The transfer function between the indicated force (i.e., the displacement, $X$ ) and the applied force, $F$, can be expressed in the frequency domain:

$$
\frac{F(\omega)}{X(\omega)}=k+i c \omega
$$

where $k$ and $c$ are the stiffness and damping of the material of the sensing unit; $\omega$ is the angular frequency; $i^{2}=-1$.

From Equation 4, the modulus of the transfer function will increase with increasing frequency, consistent with the measurements shown in Figure 8. The long-term response (i.e., static or quasi-static readings) of such a viscoelastic system depends on the stiffness, while the shortterm response (i.e., readings at high frequencies) depends on both stiffness and damping. Given that with static loads the relationship between sensor output and load is almost linear (Figure 7), the stiffness of the sensor may be assumed to be independent of the load.

\subsubsection{Load dependence}

Transfer functions between applied forces and the forces indicated by the pressure sensors were dependent on load (Figures 8 and 9). Assuming the stiffness of the sensor material is likely to be independent of load, the load dependency may arise from the damping being dependent on load. The dependence on load might also be attributed to a low pressure resolution of the sensors relative to variance in the dynamic load. With sine sweep loads, distortion with flattened peaks was noted in the time histories from some sensing units, especially with a small load (e.g., preloads of 60 and 90). A typical response containing distortion from a sensing unit is shown in Figure 13. The distortion occurred because the pressure resolution of the sensor (about $0.02 \mathrm{~N}$ in this case) was low relative to the variance in the dynamic force ( 0.10 and $0.11 \mathrm{~N}$ peak-to-peak for preloads of 60 and 90 ). 


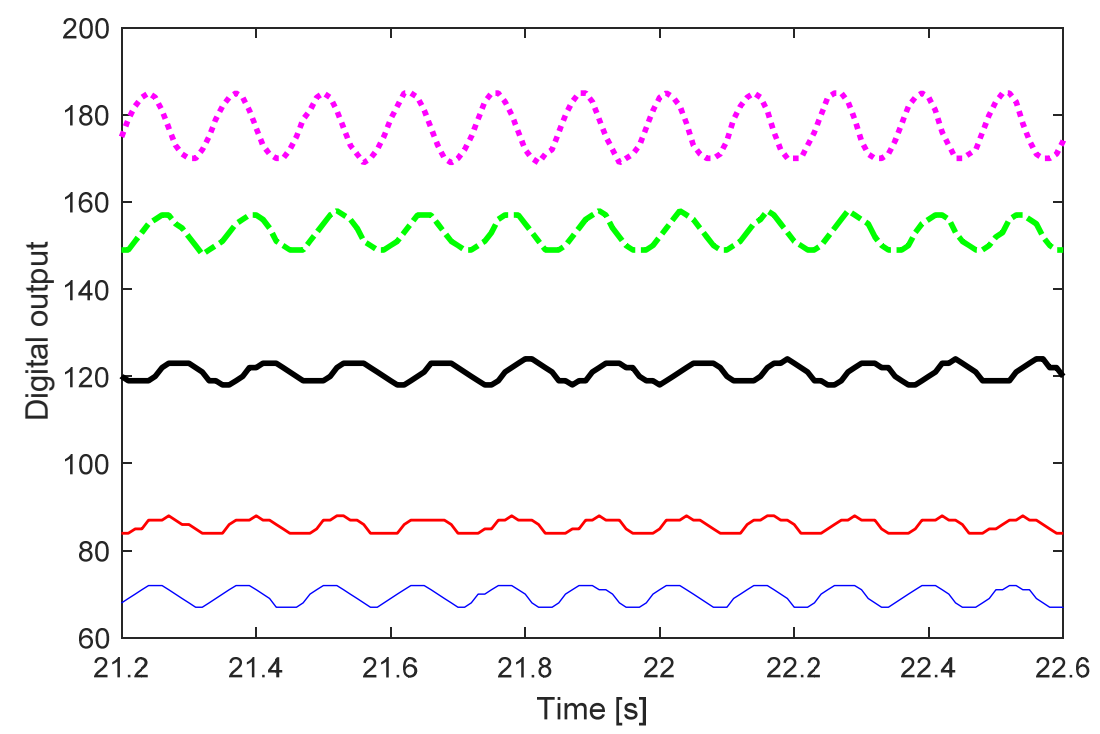

Figure 13 Measured time histories (raw digital outputs) of a sensing unit during sine sweep loads with five preloads (only a sample of data shown for clarity): — preload of 60 ; preload of $90 ;-$ preload of $120 ;-\cdot-\cdot-$ preload of $150 ; \ldots . .$. preload of 180.

\subsubsection{Effect of scanning}

Scanning introduced phase differences between the sensing units. The scanning effect was studied using a mathematical model (Figure 14). A block of mass, $m$, (30 kg) was excited with random vibration of magnitude, a, (1.0 ms-2 r.m.s.) with flat constant bandwidth acceleration spectrum between 0.5 and $15 \mathrm{~Hz}$ and a duration of $10 \mathrm{~s}$. The total force, $F_{\mathrm{t}}(t)$, associated with the motion was calculated by Equation (5). Suppose there are 2016 sensing units with a sampling rate of 100 frames per second (as with the pressure sensor investigated), the subforce, $F_{n}(t)$, was calculated by dividing the total force, $F_{t}(t)$, by 2016 (Equation 6$)$. Then each sub-force was artificially shifted by a period of time to represent the scanning procedure (Equation 7), where $F_{\mathrm{ns}}(t)$ is the shifted sub-force, and the time increment, $\mathrm{d} t$, is $4.96 \times 10^{-6} \mathrm{~s}$ (equivalent to the sampling rate of 100 frames per second over 2016 cells), $n$ is the sensing unit index. The scanned total force, $F_{\mathrm{ts}}(t)$, was calculated by summing the shifted sub-forces. The transfer function between the acceleration and the total scanned force was calculated and is referred to as the 'scanned apparent mass'. The apparent mass of a rigid block of mass is a real number equal to its mass at all frequencies. 


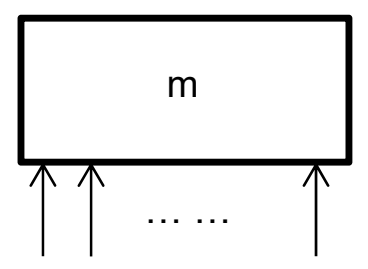

$F_{1} F_{2}$

$$
F_{\mathrm{t}}(t)=m^{*} a(t)
$$$$
F_{\mathrm{n}}(t)=F_{\mathrm{t}}(t) / 2016
$$$$
F_{\mathrm{ns}}(t)=F_{\mathrm{n}}\left(t+(n-1)^{*} \mathrm{~d} t\right)
$$

$\mathrm{F}_{2016}$
Equation (5)

Equation (6)

Equation (7)

Figure 14 Mathematical model of scanning effect.

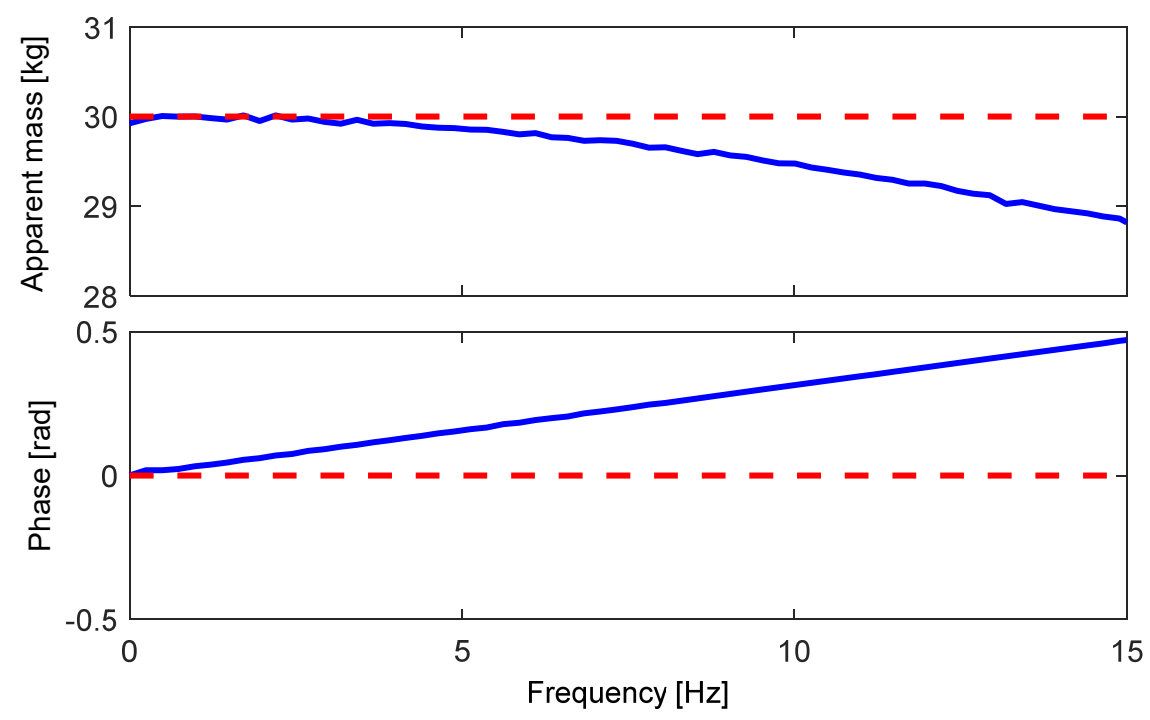

Figure 15 Comparison between the 'scanned apparent mass' and the apparent mass of the mass: effect of scanning procedure based on the mathematical model; __ 'scanned apparent mass'; - - - apparent mass of the mass.

The 'scanned apparent mass' underestimated the true dynamic force but was within $5 \%$ at all frequencies up to $15 \mathrm{~Hz}$ (Figure 15). Such underestimation is much less than that attributed to the viscoelastic behaviour of the sensor material. However, the phase error increased with increasing frequency and was as great as $0.5 \mathrm{rad}$ at $15 \mathrm{~Hz}$. A lower sampling rate over the pressure sensors would increase the errors in both the magnitude and the phase of the apparent mass.

In the calculations leading to Figure 15, the increments between each sensing unit were assumed to be equal, which differs from the real scanning procedure. The calculations illustrate the scanning effect but may not be sufficient for a quantitative compensation of the scanning errors. 


\subsection{Effectiveness of the dynamic calibration}

With the dynamic calibration applied, the errors in the forces at all the locations were reduced to less than $10 \%$ at frequencies less than $5 \mathrm{~Hz}$ and less than $30 \%$ between 5 to $15 \mathrm{~Hz}$ (Figures 11 and 12). Although when used with dynamic calibration the pressure sensors may not yield accurate forces, relative pressures between subjects can be distinguished.

One reason for dynamic calibration not eliminating errors in measuring dynamic forces at different locations of the body may be the low resolution of the pressure sensors. With a vibration magnitude of $1.0 \mathrm{~ms}^{-2}$ r.m.s., the dynamic force below the middle thighs at $5 \mathrm{~Hz}$ was around $16 \mathrm{~N}$ r.m.s. (Figure 10). With 248 loaded sensing units (each with an area of $1.03 \mathrm{~cm}^{2}$ ) beneath the middle thighs, the average dynamic pressure at each sensing unit was only about $0.73 \mathrm{kPa}$ r.m.s. The resolution of the pressure sensor was about $0.2 \mathrm{kPa}$, which was too coarse to reflect variations in dynamic pressures at the middle thighs. This explains why there were greater errors in the calibrated forces beneath the middle thighs than at other locations (Figure 11).

As the digitisation in the sensor system used 8 bits, the pressure resolution could only be increased by reducing the pressure range. However, the range is determined by the peak pressure to be measured anywhere over the pressure mat. Assuming 8-bit sampling and a maximum static pressure of $35 \mathrm{kPa}$ at the ischial tuberosities (as in this study), the sensor resolution must be coarser than $0.14 \mathrm{kPa}$, which is insufficient to measure the small variations in dynamic pressures at the middle thighs. Digitisation with more than 8-bits is likely to be required to measure dynamic pressures over the thighs.

To maximise the pressure resolution, the pressure range of the sensors should be as low as possible whilst allowing the measurement of the peak pressures caused by static and dynamic forces at the ischial tuberosities. The dynamic forces at the ischial tuberosities have been measured using load cells for both a rigid seat (Liu et al., 2017) and soft seats (Liu et al., 2016). With $1.0 \mathrm{~m} / \mathrm{s}^{2}$ r.m.s. vertical random vibration having a constant bandwidth acceleration power spectrum between 0.25 and $20 \mathrm{~Hz}$, at the $5-\mathrm{Hz}$ principal resonance in the apparent mass of the body the mean ratio between the peak dynamic force and the static force was about 1.17 with the rigid seat and 1.15 with soft seats. A multiplying factor greater than 1.2 should therefore be used to estimate the peak dynamic pressures from the peak static pressures when identifying the pressure range of the sensor. The ratio will be greater than 1.2 for some subjects and will increase with increases in the magnitude of the vibration.

With dynamic loads applied by compressing foam, the moduli of the transfer functions between the applied forces and the outputs of the sensors were greater for sensor units at the centre 
of the foam than those at the edges of the foam (Figure 9), showing that dynamic pressures beneath the foam were greater at the edges. The non-uniformity in dynamic pressures may be caused by properties of the foam including air flow within the foam. The dynamic compression of a foam block therefore seems unsuitable for the dynamic calibration of individual sensing units. To calibrate each sensing unit, a device is required that can produce dynamic pressures evenly over a defined area of the pressure mat. A non-compressible fluid such as water contained within a membrane might be suitable if the membrane is sufficiently flexible to not alter the pressures applied to the surfaces of the sensors while being sufficiently inelastic to not expand during cyclical loading.

Although the measurements reported in this paper were obtained using resistive pressure sensors, errors in the measurement of dynamic pressures have also been reported when using a capacitive pressure mat (Hinz, et al., 2006). With capacitive pressure sensors, errors can arise from the viscoelastic behaviour of the material placed between the two plates of a condenser and from the low resolution of pressures when using 8-bit digitisation. Although the cause for the viscoelastic behaviour can differ between capacitive sensors and resistive sensors, the calibration method designed in this study may be used for the dynamic calibration of groups of capacitive sensors.

\subsection{Recommendations}

The measurement of dynamic pressures requires consideration of the dynamic performance of the pressure sensors, factors affecting the dynamic performance of the sensors, and the distribution of pressures to be measured. When measuring dynamic pressures between the human body and a seat during whole-body vibration it is suggested:

- With common magnitudes of vibration, the static pressures between the human body and a seat are much greater than the variations in dynamic pressures during wholebody vibration (see Section 4.3). Measurements of dynamic pressures require a finer resolution than measurements of static pressures: digitisation with more than 8-bits is likely to be required, and the pressure range of the sensors should be as low as possible whilst able to measure the peak dynamic pressures.

- Dynamic pressures beneath the ischial tuberosities are much greater than those beneath the middle thighs, especially with high stiffness seats, so different sensitivities are desirable when measuring dynamic pressures at the ischial tuberosities and the middle thighs, even though the same sensitivity can be used when measuring static pressures. 
- The maximum measurable pressure should be greater than the sum of the static pressure and the peak dynamic pressure at the resonance frequency beneath the ischial tuberosities. With a vibration magnitude of $1.0 \mathrm{~ms}^{-2}$ r.m.s., the peak dynamic pressure will be greater than 1.2 times the maximum static pressure.

- The noise floor should be lower than the lowest pressure to be measured (e.g., beneath the middle thighs at low or high frequencies).

- Prior to a static calibration, the pressure sensors should be loaded for at least 1 minute (or other period if found to be appropriate).

- The gains of the pressure sensors measured during a static calibration can be used for static calibration of individual pressure sensing units.

- The gains of pressure sensors measured during dynamic calibration can be used for dynamic calibration of groups of pressure sensors.

- Calibrations should be repeated before and after dynamic measurements to check that the sensors remained in the same condition.

- Wrinkles in a pressure mat caused by deflection of a soft seat during loading, or the curved surface of a rigid seat, can give rise to artefacts in pressure measurements.

\section{Conclusions}

Without dynamic calibration, the total dynamic force indicated by a resistive pressure mat was underestimated by $30 \%$ to $42 \%$ at frequencies between 1 and $15 \mathrm{~Hz}$. Underestimations of dynamic forces may be caused by the viscoelastic properties of the sensor material, low pressure resolution, and a scanning effect, with the viscoelastic behaviour the principal factor. Underestimation due to low pressure resolution depends on the magnitude of the dynamic forces relative to the sensitivity. At frequencies up to $15 \mathrm{~Hz}$, the scanning effect caused less than $5 \%$ underestimation in the measured forces, but had a greater effect on the phase.

A method of calibrating a pressure mat by applying controlled static and dynamic forces to the pressure sensors through a foam block is proposed. The method can provide reasonable static calibration of individual sensing units and dynamic calibration to reduce errors in the total forces summed over pressure sensors. However, the dynamic calibration of individual pressure sensors is limited by non-uniformity in the pressures beneath the foam during dynamic loads. It has been shown that the calibration method can reduce errors in dynamic forces at the ischial tuberosities, the middle thighs, and the front thighs during vertical wholebody vibration to less than $10 \%$ at frequencies less than $5 \mathrm{~Hz}$ and to less than $30 \%$ at frequencies between 5 and $15 \mathrm{~Hz}$. 


\section{References}

Arndt, A., 2003. Correction for sensor creep in the evaluation of long-term plantar pressure data. Journal of Biomechanics, 36 (12), 1813-1817.

Dewangan, K., Rakheja, S., Marcotte, P. and Shahmir, A., 2013. Effects of elastic seats on seated body apparent mass responses to vertical whole body vibration. Ergonomics, 58 (7), $1-30$.

Ebe, K., Griffin, M.J., 2000a. Qualitative models of seat discomfort including static and dynamic factors. Ergonomics, 43 (6), 771-790.

Ebe, K., Griffin, M.J., 2000b. Quantitative prediction of overall seat discomfort. Ergonomics, 43 (6), 791-806.

Ebe, K., and Griffin, M.J., 2001. Factors effecting static and seat cushion comfort. Ergonomics, 41 (10), 901-921.

Fairley, T.E., and Griffin, M.J., 1989. The apparent mass of the seated human body: vertical vibration. Journal of Biomechanics, 22 (2), 81-94.

Liu, C., Qiu, Y., Griffin, M.J., 2016. On the apparent mass of the human body sitting on a soft seat with vertical vibration. The $51^{\text {st }}$ United Kingdom Conference on Human Responses to Vibration, Gosport, England.

Liu, C., Qiu, Y., Griffin, M.J., 2017. Dynamic forces over the interface between a seated human body and a rigid seat during vertical whole-body vibration. Journal of Biomechanics, 61 (16) 176-182.

Hinz, B., Rutzel, S., Bluthner, R., Menzel, G., Wöfel, H.P., Seidel, H., 2006. Apparent mass of seated man - First determination with a soft seat and dynamic seat pressure distributions. Journal of Sound and Vibration, 298 (3), 704-724.

Palmer, M.C., O'Rourke, T.D., Olson, N.A., Abdoun, T., Ha, D., O’Rourke, M.J., 2009. Tactile pressure sensors for soil-structure interaction assessment. Journal of Geotechnical and Geoenvironmental Engineering, ASCE, Vol. November, 1638-1645.

Paikowsky, S.G., Hajduk, E.L., 1997. Calibration and use of grid based tactile pressure sensors in granular material. Geotechnical Testing Journal, 20, 218-241. 
Saggin, B., Scaccabarozzi, D., Tarabini, M., 2013. Metrological performances of a plantar pressure measurement system. IEEE transactions on instrumentation and measurement, 62 (4), 766-776.

Tarabini, M., Saggin, B., Scaccabarozzi, D., Gaviraghi, D., Moschioni, G., 2013. Apparent mass distribution at the feet of standing subjects exposed to whole-body vibration. Ergonomics 56 (5), 842-855.

Wu, X., Rakheja, S., Boileau, P.É., 1998. Study of human-seat interface pressure distribution under vertical vibration. International Journal of Industrial Ergonomics, 21, 433-449.

Wu, X., Rakheja, S., Boileau, P.É., 1999. Distribution of human-seat interface pressure on a soft automotive seat under vertical vibration. International Journal of Industrial Ergonomics, 24 (5), 545-557.

Zhou, Z., Griffin, M.J., 2014. Response of the seated human body to whole-body vertical vibration: discomfort caused by sinusoidal vibration. Ergonomics, 57 (5), 714-732. 